\title{
Adjuvant Therapy for Resected Biliary Tract Cancer: ASCO Clinical Practice Guideline
}

Rachna T. Shroff, MD ${ }^{1}$; Erin B. Kennedy, MHSc${ }^{2}$; Melinda Bachini ${ }^{3}$; Tanios Bekaii-Saab, MD ${ }^{4}$; Christopher Crane, MD $^{5}$; Julien Edeline, MD, PhD ${ }^{6}$; Anthony El-Khoueiry, MD; Mary Feng, MD ${ }^{8}$; Matthew H.G. Katz, MD ${ }^{9}$; John Primrose, MD ${ }^{10}$; Heloisa P. Soares, MD, PhD ${ }^{11}$; Juan Valle, MD ${ }^{12}$; and Shishir K. Maithel, MD ${ }^{13}$

ASSOCIATED

CONTENT

Appendix

Data Supplement

Author affiliations

and support

information (if

applicable) appear

at the end of this $\sim$

article.

Accepted on

December 12, 2018 ? and published at jco.

org on March 11,

2019: DOI https://doi.

org/10.1200/JC0.18.

02178

R.T.S. and S.K.M.

were Expert Panel

co-chairs.

Clinical Practice

Guidelines

Committee approval:

October 9, 2018

Editor's note: This

American Society of

Clinical Oncology

Clinical Practice

Guideline provides

recommendations,

with comprehensive

review and analyses

of the relevant

literature for each

recommendation.

Additional

information,

including a Data

Supplement with

additional evidence

tables, slide sets,

clinical tools and

resources, and links

to patient

information at www.

cancer.net, is

available at www.

asco.org/

gastrointestinal-

cancer-guidelines.

Reprint Requests:

2318 Mill Rd, Ste

800, Alexandria, VA

22314; guidelines@

asco.org

PURPOSE To develop an evidence-based clinical practice guideline to assist in clinical decision making for patients with resected biliary tract cancer.

METHODS ASCO convened an Expert Panel to conduct a systematic review of the literature on adjuvant therapy for resected biliary tract cancer and provide recommended care options for this patient population.

RESULTS Three phase III randomized controlled trials, one phase II trial, and 16 retrospective studies met the inclusion criteria.

RECOMMENDATIONS Based on evidence from a phase III randomized controlled trial, patients with resected biliary tract cancer should be offered adjuvant capecitabine chemotherapy for a duration of 6 months. The dosing used in this trial is described in the qualifying statements, while it should be noted that the dose of capecitabine may also be determined by institutional and regional practices. Patients with extrahepatic cholangiocarcinoma or gallbladder cancer and a microscopically positive surgical resection margin (R1 resection) may be offered chemoradiation therapy. A shared decision-making approach is recommended, considering the risk of harm and potential for benefit associated with radiation therapy for patients with extrahepatic cholangiocarcinoma or gallbladder cancer. Additional information is available at www.asco.org/ gastrointestinal-cancer-guidelines.

J Clin Oncol 37. $\odot 2019$ by American Society of Clinical Oncology

\section{INTRODUCTION}

Biliary tract cancers include cancer of the intrahepatic bile ducts, perihilar and distal extrahepatic bile ducts, and the gallbladder. There were an estimated 12,190 new diagnoses and 3,790 deaths from gallbladder and extrahepatic bile duct cancer in the United States in 2018. ${ }^{1}$ Five-year relative survival rates range from $2 \%$ to $15 \%$ and from $2 \%$ to $30 \%$ for intrahepatic and extrahepatic cholangiocarcinoma, respectively, ${ }^{2}$ while the 5-year relative survival rate for gallbladder cancer ranges from $2 \%$ to $70 \%$, depending on stage. ${ }^{3}$ Hilar tumors account for approximately $60 \%$ to $70 \%$ of cholangiocarcinomas. ${ }^{4}$ Gallbladder cancer is the most common site of biliary tract cancer and is also associated with shorter time to recurrence and survival time after recurrence than hilar cholangiocarcinoma. ${ }^{5} \mathrm{Bil}-$ iary tract cancers are relatively rare in the Western world; however, higher incidence clusters have been found in some areas of Asia and the Andes. ${ }^{6}$

Biliary tract cancers usually present at an advanced stage, and only approximately $20 \%$ of tumors are considered resectable. ${ }^{7}$ Surgery is the primary curative treatment option for early-stage biliary tract cancer; however, due to the high rates of recurrence with resection alone, there remains a need for effective adjuvant therapy to improve rates of relapse-free survival (RFS) and overall survival (OS), while maintaining health-related quality of life. ${ }^{8}$

Studies of adjuvant treatment options have historically been small, retrospective, and nonrandomized and include a mix of patients with gallbladder and other biliary tract tumors. ${ }^{9}$ In addition, recommendations from clinical practice guidelines have largely been consensus based. ${ }^{5,10,11}$ Recently, results from prospective trials of adjuvant therapy for biliary tract cancers have been published, including randomized controlled comparisons of chemotherapy options compared with observation and a prospective single-arm trial of chemoradiation. The purpose of this guideline development project is to review this newer evidence and provide evidence-based recommendations for adjuvant therapy for patients with resected biliary tract cancer, as well as to consider recommendations for patients who are at higher risk for microscopically positive resection margins. 


\section{THE BOTTOM LINE}

\section{Adjuvant Therapy for Resected Biliary Tract Cancer: ASCO Clinical Practice Guideline}

\section{Guideline Question}

Is adjuvant therapy, including fluoropyrimidine-based or gemcitabine-based chemotherapy and/or radiation therapy, recommended for patients with resected biliary tract cancer?

\section{Target Population}

Patients with resected biliary tract cancer.

\section{Target Audience}

Oncologists (medical, radiation, and surgical) and other health care professionals who treat patients with biliary tract cancer, patients, and caregivers.

\section{Methods}

An Expert Panel was convened to develop clinical practice guideline recommendations based on a systematic review of the medical literature.

\section{Recommendations}

Recommendation 1. Patients with resected biliary tract cancer should be offered adjuvant capecitabine chemotherapy for a duration of 6 months (Type: Evidence based; Benefits outweigh harms; Evidence quality: Intermediate; Strength of recommendation: Moderate).

\section{Qualifying statements.}

- In the BILCAP (Adjuvant Capecitabine for Biliary Tract Cancer) phase III randomized controlled trial, capecitabine was delivered at a dose of $1,250 \mathrm{mg} / \mathrm{m}^{2}$ twice a day on treatment days 1 to 14 of a 3-week cycle for 24 weeks (eight cycles). ${ }^{12}$

- The Expert Panel agrees that the recommended dose of capecitabine may be determined by institutional and regional practices.

Recommendation 2. Patients with extrahepatic cholangiocarcinoma or gallbladder cancer and a microscopically positive surgical margin resection (R1 resection) may be offered chemoradiotherapy (Type: Evidence and Consensus based; Benefits outweigh harms; Evidence quality: Low; Strength of recommendation: Moderate).

\section{Qualifying statements.}

- A shared decision-making approach is recommended, considering the risk of potential harm and potential for benefit associated with radiation therapy for patients with extrahepatic cholangiocarcinoma or gallbladder cancer.

- The Expert Panel notes that in the SWOG0809 prospective single-arm trial of chemoradiotherapy, ${ }^{13}$ radiation was delivered at a dose of 45 Gy to regional lymphatics and 54 to 59.4 Gy to the tumor bed. However, at this time, the evidence base is not sufficiently well developed to make a recommendation for optimal dosing of radiation therapy in the context of chemoradiation therapy.

\section{Additional Resources}

More information, including a Data Supplement with additional evidence tables, slide sets, and clinical tools and resources, is available at www.asco.org/gastrointestinal-cancer-guidelines. The Methodology Manual (available at www.asco.org/guideline-methodology) provides additional information about the methods used to develop this guideline. Patient information is available at www.cancer.net.

ASCO believes that cancer clinical trials are vital to inform medical decisions and improve cancer care, and that all patients should have the opportunity to participate.

\section{GUIDELINE QUESTION}

This clinical practice guideline addresses the following clinical question: Is adjuvant therapy, including fluoropyrimidinebased or gemcitabine-based chemotherapy and/or radiation therapy, recommended for patients with resected biliary tract cancer?

\section{METHODS}

\section{Guideline Development Process}

This systematic review-based guideline was developed by a multidisciplinary Expert Panel, which included members with expertise in surgical oncology, medical oncology, radiation oncology, practice guideline implementation, and 
health research methodology, as well as a member representing the patient perspective. The Expert Panel (Appendix Table A1, online only) met via teleconference and/or webinar and corresponded through e-mail. Based on consideration of the evidence, the authors were asked to contribute to the development of the guideline, provide critical review, and finalize the guideline recommendations. The guideline recommendations were sent for an open comment period of 2 weeks, allowing the public to review and comment on the recommendations after submitting a confidentiality agreement. These comments were taken into consideration while finalizing the recommendations. Members of the Expert Panel were responsible for reviewing and approving the penultimate version of the guideline, which was then submitted to Journal of Clinical Oncology for editorial review and consideration for publication. All ASCO guidelines are ultimately reviewed and approved by the Expert Panel and the ASCO Clinical Practice Guidelines Committee before publication. All funding for the administration of the project was provided by ASCO.

PubMed was systematically searched from January 2008 to June 30,2018 , for full-text publications of randomized controlled trials, prospective controlled studies, or singlearm trials. Details of the search strategy are provided in the Data Supplement. Articles were selected for inclusion if they were studies of adjuvant chemotherapy and/or radiotherapy for patients with resected biliary tract cancer. A search of recent abstracts (2017 to 2018) from the ASCO Annual Meeting and the ASCO Gastrointestinal Cancers Symposium was also conducted for the article types mentioned previously.

Resection margins are classified as RO (negative margins), R1 (positive microscopic margins), or R2 (positive macroscopic margins). ${ }^{14}$ Margin status is highly correlated with local recurrence and survival. ${ }^{11}$ The Expert Panel recognized that the subset of patients with hilar extrahepatic cholangiocarcinoma have a higher likelihood of microscopically positive (R1) resection margins than patients with other disease sites due to the central location of the tumor, ${ }^{15}$ and the panel chose to extend the search in this patient population to include retrospective studies from January 1, 2004, to June 30, 2018. Abstracts were not considered for potential inclusion in this extended search. Reference lists of systematic reviews found in the PubMed search were used as a source of potential articles for inclusion. Outcomes of interest included OS, RFS, adverse events, and quality of life. Articles were excluded from the systematic review if they were editorials, commentaries, letters, news articles, case reports, narrative reviews, or if they were published in a non-English language.

The guideline recommendations were crafted, in part, using the Guidelines Into Decision Support methodology and accompanying BRIDGE-Wiz software (Yale University, New Haven, CT).${ }^{16}$ In addition, a guideline implementability review was conducted. Based on the implementability review, revisions were made to the draft to clarify recommended actions for clinical practice. Ratings for the type and strength of recommendation, evidence, and potential bias are provided with each recommendation.

\section{Study Quality Assessment}

Risk of bias in the phase III randomized controlled trials was assessed using the Cochrane Risk of Bias Tool. ${ }^{17}$ Additional quality domains, including imprecision, inconsistency, indirectness, and potential for publication bias, were also assessed. ${ }^{18} \mathrm{~A}$ risk-of-bias assessment of the SWOG0809 trial was conducted using the Risk of Bias in Nonobservational Studies of Intervention tool. ${ }^{19}$ The retrospective observational studies included in this review were rated using guidance contained within the Cochrane Handbook for Systematic Reviews of Interventions (Section 12.2.1), ${ }^{18}$ which generally rates observational studies as low quality due to issues of residual confounding and selection bias inherent to nonrandomized study designs. However, observational studies may be rated as higher quality if the effect size is large and there is no obvious bias explaining those effects, when there is an apparent doseresponse gradient, or when residual confounding after adjusted analysis would be expected to lessen the likelihood of a significant result. ${ }^{18}$

Further information about the methods used to develop this guideline is available in the Methodology Manual at www. asco.org/guideline-methodology, including an overview (eg, panel composition, development process, and revision dates), literature search and data extraction, the recommendation development process (Guidelines Into Decision Support and BRIDGE-Wiz), and quality assessment.

The ASCO Expert Panel and guidelines staff will work with co-chairs to keep abreast of any substantive updates to the guideline. ASCO uses a signals approach to updating, which is designed to identify only new, potentially practicechanging data - signals - that might translate into revised practice recommendations. ${ }^{20}$ The approach relies on targeted routine literature searching and the expertise of ASCO Expert Panel members to help identify potential signals. The Methodology Manual (available at www.asco. org/guideline-methodology) provides additional information about the methods used to develop this guideline. This is the most recent information as of the publication date.

\section{Guideline Disclaimer}

The Clinical Practice Guidelines and other guidance published herein are provided by the American Society of Clinical Oncology, Inc. (ASCO) to assist providers in clinical decision making. The information herein should not be relied upon as being complete or accurate, nor should it be considered as inclusive of all proper treatments or methods of care or as a statement of the standard of care. With the rapid development of scientific knowledge, new evidence may emerge between the time information is developed 
and when it is published or read. The information is not continually updated and may not reflect the most recent evidence. The information addresses only the topics specifically identified therein and is not applicable to other interventions, diseases, or stages of diseases. This information does not mandate any particular course of medical care. Further, the information is not intended to substitute for the independent professional judgment of the treating provider, as the information does not account for individual variation among patients. Recommendations reflect high, moderate, or low confidence that the recommendation reflects the net effect of a given course of action. The use of words like "must," "must not," "should," and "should not" indicates that a course of action is recommended or not recommended for either most or many patients, but there is latitude for the treating physician to select other courses of action in individual cases. In all cases, the selected course of action should be considered by the treating provider in the context of treating the individual patient. Use of the information is voluntary. ASCO provides this information on an "as is" basis and makes no warranty, express or implied, regarding the information. ASCO specifically disclaims any warranties of merchantability or fitness for a particular use or purpose. ASCO assumes no responsibility for any injury or damage to persons or property arising out of or related to any use of this information, or for any errors or omissions.

\section{Guideline and Conflicts of Interest}

The Expert Panel was assembled in accordance with ASCO's Conflict of Interest Policy Implementation for Clinical Practice Guidelines ("Policy," found at http://www. asco.org/rwc). All members of the Expert Panel completed ASCO's disclosure form, which requires disclosure of financial and other interests, including relationships with commercial entities that are reasonably likely to experience direct regulatory or commercial impact as a result of promulgation of the guideline. Categories for disclosure include employment; leadership; stock or other ownership; honoraria, consulting or advisory role; speaker's bureau; research funding; patents, royalties, other intellectual property; expert testimony; travel, accommodations, expenses; and other relationships. In accordance with the Policy, the majority of the members of the Expert Panel did not disclose any relationships constituting a conflict under the Policy.

\section{RESULTS}

\section{Summary of Results}

Four prospective studies met the inclusion criteria for this review, including three phase III randomized controlled trials of chemotherapy compared with observation ${ }^{12,21,22}$ and a prospective single-arm trial of chemoradiation. ${ }^{13}$ Study characteristics, including chemotherapy dose and schedule information, are listed in Table 1. RFS, median and 5-year OS, pattern of relapse, quality of life, and adverse events were extracted where reported and are listed in Tables 2 to 4 . Further description of each of these prospective trials is included in Recommendations. In addition, 16 retrospective studies that included data for patients with hilar cholangiocarcinoma were included in the evidence base. The characteristics and outcomes of these studies are further described under Recommendation 2 and within Data Supplement Tables 1 to 4.

\section{Quality Assessment of the Literature (Data Supplement Tables 5 and 6)}

Randomized controlled trials. The results across the three trials of chemotherapy compared with observation and surveillance included one positive ${ }^{12}$ and two negative trials. ${ }^{21,22}$ All phase III trials were found to be at risk for bias due to lack of blinding of study participants and personnel; however, this risk would have been less serious for the outcome of OS. ${ }^{12,21,22}$ Patient characteristics were well balanced between study arms across trials. Results were provided for the intent-to-treat population. ${ }^{12,21,22}$

The study by Ebata et $a^{21}$ failed to accrue the planned number of patients, which could have resulted in an underpowered analysis. However, the authors of this study note that this factor was unlikely to have affected the final results. The PRODIGE 12 trial had significant imprecision around the estimate for the primary outcome of RFS. ${ }^{22}$ Evidence quality (ie, certainty) for the comparisons of gemcitabine plus oxaliplatin or gemcitabine alone versus observation was judged to be low due to these limitations. The survival outcomes for the comparison of capecitabine versus observation (BILCAP [Adjuvant Capecitabine for Biliary Tract Cancer] $)^{12}$ were rated as intermediate quality as a result of the significant magnitude of the OS effect in the per-protocol and prespecified adjusted ITT analyses.

Nonrandomized studies. The remainder of the studies were retrospective observational studies rated as low quality due to issues of residual confounding and selection bias inherent to nonrandomized study designs. ${ }^{18}$ The phase II SWOG0809 single-arm trial included patients with a higher rate of RO resections than expected based on historical data. ${ }^{13}$ For the studies that included patients with hilar cholangiocarcinoma, ${ }^{21,23-37}$ methodologic or quality issues included inconsistency of intervention and included populations across some studies, as well as smaller sample sizes within the studies that were conducted at single institutions. Risk of bias due to confounding was reduced in some studies by adjusting for prognostic factors or by matching patients according to their likelihood of receiving adjuvant therapy.

\section{RECOMMENDATIONS}

\section{Clinical Question}

Is adjuvant therapy, including fluoropyrimidine-based or gemcitabine-based chemotherapy and/or radiation therapy, recommended for patients with resected biliary tract cancer? 


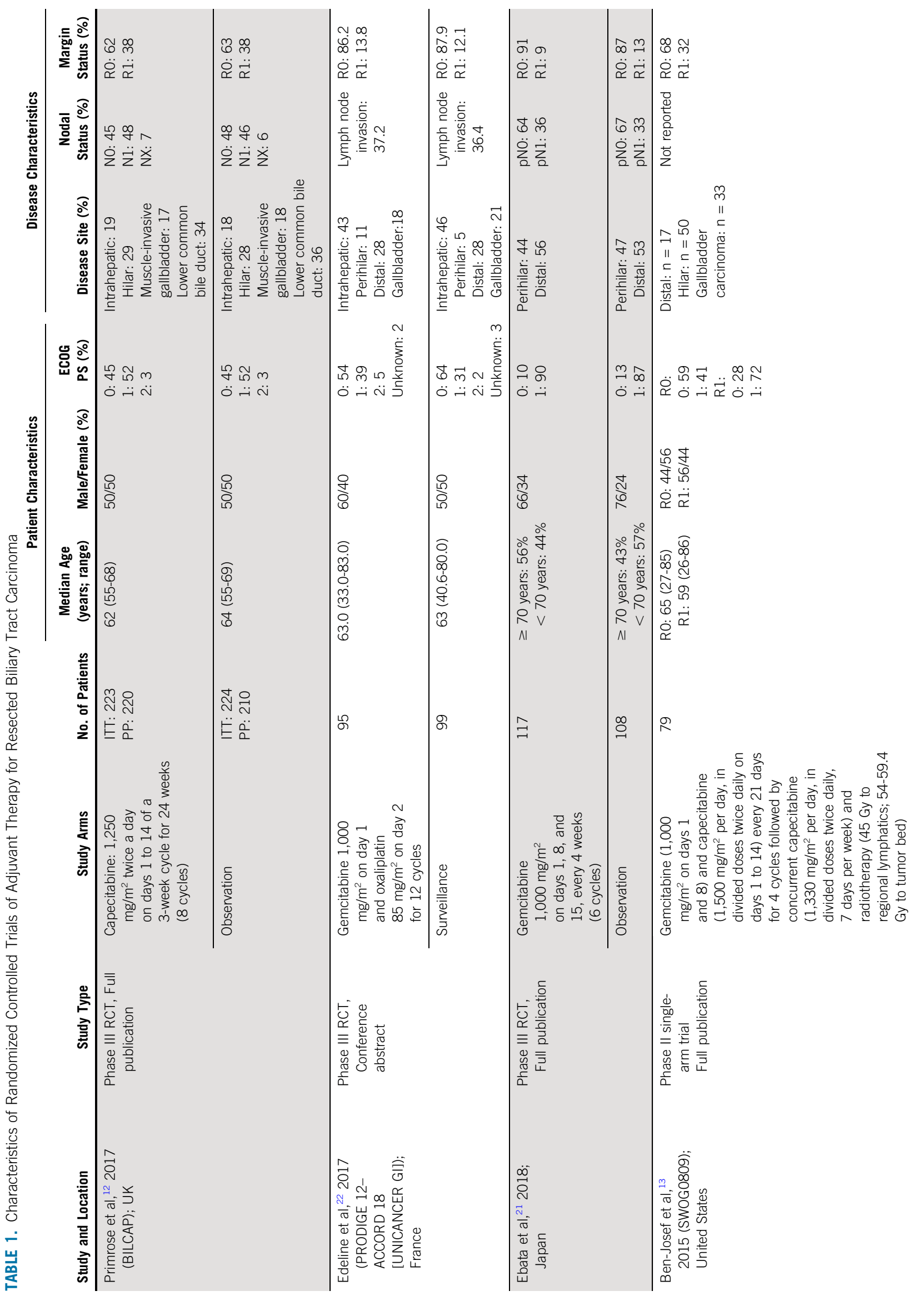

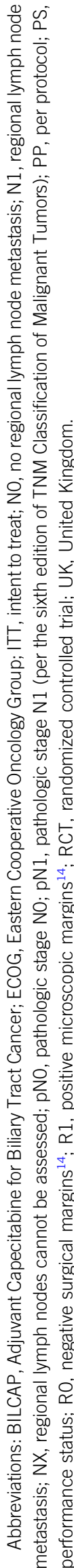




\section{Recommendation 1}

Patients with resected biliary tract cancer should be offered adjuvant capecitabine chemotherapy for a duration of 6 months (Type: Evidence-based; Benefits outweigh harms; Evidence quality: Intermediate; Strength of recommendation: Moderate).

\section{Qualifying statements.}

- In the BILCAP phase III randomized controlled trial, capecitabine was delivered at a dose of $1,250 \mathrm{mg} / \mathrm{m}^{2}$ twice a day on treatment days 1 to 14 of a 3-week cycle for 24 weeks (eight cycles). ${ }^{12}$

- The Expert Panel agrees that the recommended dose of capecitabine may be determined by institutional and regional practices.

Literature review and analysis. BILCAP (United Kingdom). The BILCAP study ${ }^{12}$ included 447 patients with intrahepatic cholangiocarcinoma (19\%), hilar cholangiocarcinoma (29\%), muscle-invasive gallbladder cancer (18\%), or cholangiocarcinoma of the lower common bile duct (35\%). The percentages of patients reported to have involved lymph nodes in this trial in the treatment and control groups were $45 \%$ and $48 \%$, respectively. The rate of $\mathrm{R} 1$ resection was $38 \%$ in both the treatment and control groups. The study included patients with Eastern Cooperative Oncology Group (ECOG) performance status (PS) of 0 to 2 who had undergone $\mathrm{R} 0$ or $\mathrm{R} 1$ resections.

This study found a significant difference in OS in a prespecified ITT analysis adjusted for nodal status, disease grade, and sex (hazard ratio [HR], 0.71; $95 \% \mathrm{Cl}, 0.55$ to 0.92; $P<.01$ ). A per-protocol analysis also found a significant difference in OS (HR, $0.75 ; 95 \% \mathrm{Cl}, 0.58$ to 0.97 ; $P=.028$ ) in favor of capecitabine versus observation, while there was no significant difference in unadjusted ITT OS (HR, 0.81; 95\% Cl, 0.63 to $1.04 ; P=.097$ ). RFS also significantly favored the experimental group in this study ( $\mathrm{HR}, 0.71 ; 95 \% \mathrm{Cl}, 0.54$ to 0.92; $P=.001$; Table 2). There were no significant differences in quality of life, and the most common adverse event in the capecitabine group was palmar-plantar erythema (21\%; Table 4).

PRODIGE 12 (France). The PRODIGE 12 study $^{22}$ included 194 patients with intrahepatic $(46 \%)$, perihilar $(8 \%)$, or distal $(27 \%)$ cholangiocarcinoma or gallbladder adenocarcinoma (20\%). The percentage of patients with involved lymph nodes was $63 \%$ in the gemcitabine and oxaliplatin group and $64 \%$ in the surveillance group. The $\mathrm{R} 0$ resection rates in the treatment and control groups were $86 \%$ and $88 \%$, respectively, while the remainder were $\mathrm{R} 1$ resections. The ECOG PS for patients ranged from 0 to 2 .

No significant differences between the gemcitabine and oxaliplatin and surveillance groups were found for the primary outcomes in this study, including RFS $(\mathrm{HR}, 0.88 ; 95 \% \mathrm{Cl}$, 0.62 to $1.25 ; P=.48$ ) and global health-related quality-of-life scores at 12 months (70.8 $v 83.3$, respectively; $P=.18$ ) and 24 months (75.0 v 83.3, respectively; $P=.50$ ).
Ebata et al (Japan). This study included patients with extrahepatic cholangiocarcinoma (45\% hilar, 55\% distal) and ECOG PS of 0 or 1 . The percentages of patients reported to have involved lymph nodes in the treatment and control groups were $64 \%$ and $67 \%$, respectively. The $\mathrm{R} 0$ resection rates in the treatment and control groups were $91 \%$ and $87 \%$, respectively, while the remainder were $\mathrm{R} 1$ resections. There was no significant difference in RFS (HR, 0.93; 95\% $\mathrm{Cl}, 0.66$ to $1.32 ; P=.693$ ) or OS (HR, $1.01 ; 95 \% \mathrm{Cl}, 0.70$ to $1.45 ; P=.964)$ in this study.

Clinical interpretation. Based on the significant results of the BILCAP trial, the Expert Panel recommends that capecitabine for a period of 6 months should be offered as adjuvant therapy to patients with resected biliary tract cancer. No high-level evidence was found to warrant the recommendation of radiation therapy alone for patients with biliary tract cancer.

\section{Recommendation 2}

Patients with extrahepatic cholangiocarcinoma or gallbladder cancer and a microscopically positive surgical margin resection (R1 resection) may be offered chemoradiation therapy (Type: Evidence and Consensus based; Benefits outweigh harms; Evidence quality: low; Strength of recommendation: Moderate).

\section{Qualifying statements.}

- A shared decision-making approach is recommended, considering the potential risk of harm and potential for benefit associated with radiation therapy for patients with extrahepatic cholangiocarcinoma or gallbladder cancer.

- The Expert Panel notes that in the SWOG0809 prospective single-arm trial of chemoradiotherapy, ${ }^{13}$ radiation was delivered at a dose of 45 Gy to regional lymphatics and 54 to 59.4 Gy to the tumor bed. However, at this time, the evidence base is not sufficiently well developed to make a recommendation for optimal dosing of radiation therapy in the context of chemoradiation therapy.

Literature review and analysis. Sixteen nonrandomized studies provided data for outcomes of adjuvant therapy in patients with hilar cholangiocarcinoma, combined hilar and/or distal cholangiocarcinoma, and/or gallbladder cancer (Data Supplement). ${ }^{21,23-37}$ Because this group of studies was rated low quality, the Expert Panel largely relied on informal consensus for the development of Recommendation 2. Limited supportive evidence for this recommendation within the 16 studies includes the finding of a significant positive association between OS and adjuvant external-beam radiotherapy in the R1/R2 subgroup, but not in the RO subgroup, in the single-institution study of patients with hilar cholangiocarcinoma by Cheng et al. ${ }^{25}$ In addition, a significant positive association between RFS and OS and any adjuvant therapy was found in the 
TABLE 2. Outcomes of Randomized Controlled Trials of Adjuvant Therapy for Resected Biliary Tract Carcinoma

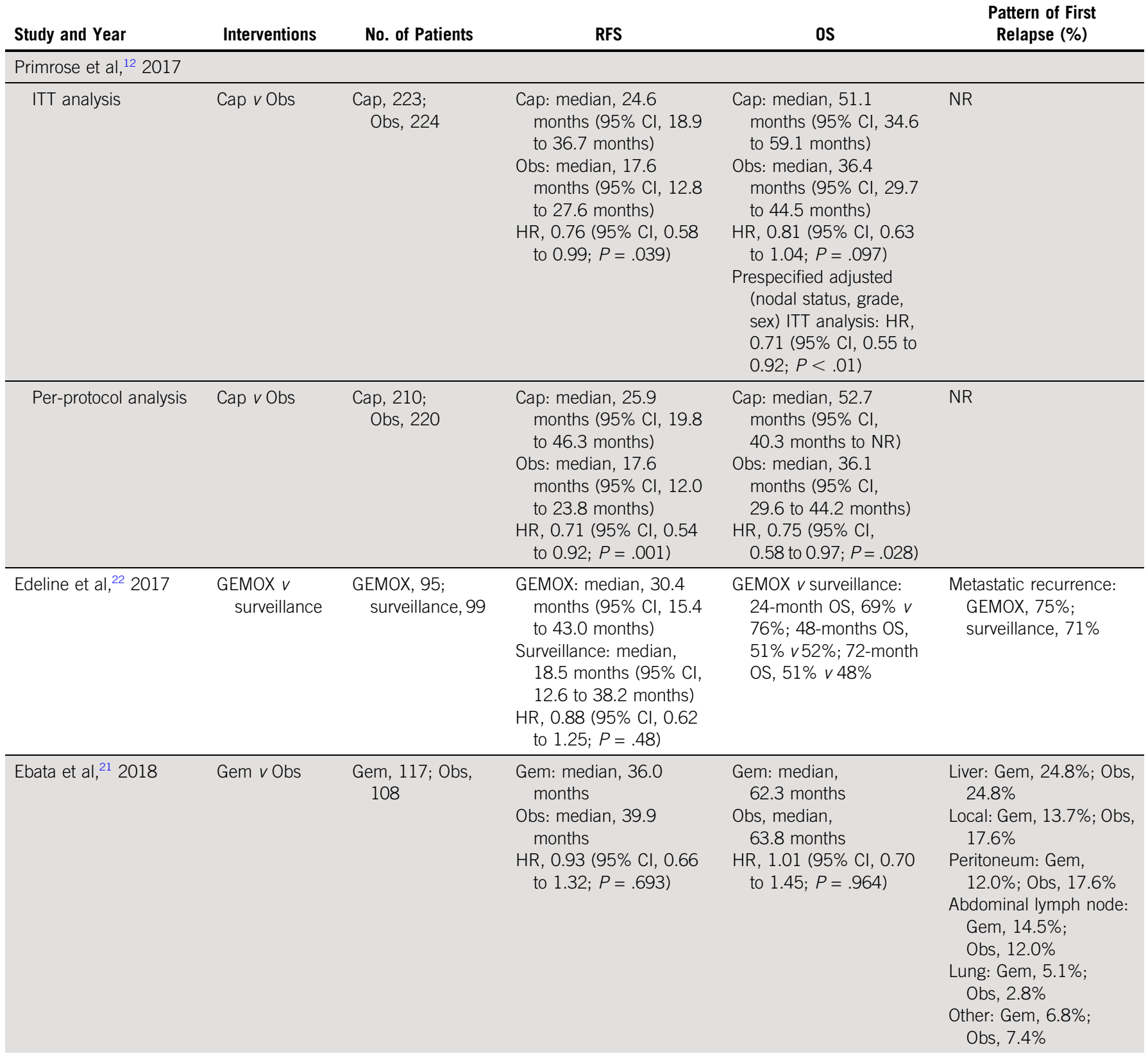

Abbreviations: Cap, capecitabine; Gem, gemcitabine; GEMOX, gemcitabine and oxaliplatin; HR, hazard ratio; ITT, intent-to-treat; NR, not reported; Obs, observation; OS, overall survival; RFS, relapse-free survival.

multi-institution study of patients with hilar cholangiocarcinoma by Krasnick et al, ${ }^{30}$ in which $40 \%$ of treated patients had an R1 resection.

Because of the limited and inconsistent outcomes data available for patients with hilar cholangiocarcinoma, the Expert Panel also discussed the relevance of included studies that provided data for other biliary tract subsites. This included the SWOG0809 phase II study of chemoradiation (Table 1 provides dose and timing) that reported outcomes for 79 patients with hilar cholangiocarcinoma (48\%), distal cholangiocarcinoma (16\%), or gallbladder cancer (32\%). ${ }^{13}$ The authors of this study found a similar rate of local recurrence and median OS in the RO and R1 subgroups, where poorer outcomes in the R1 group would have been expected based on historical data. They cautiously attribute the efficacy of adjuvant therapy in R1 patients in this study to the positive effect of treatment. ${ }^{13}$ Other supporting data for Recommendation 2 include a meta-analysis by Horgan et al, ${ }^{8}$ which included 20 studies from 1960 to 2010 and contained a similar evidence base as this review. In an analysis by margin status, the authors found a significant benefit of adjuvant therapy in patients 
TABLE 3. Outcomes for the SWOG0809 Phase II Trial of CRT

\begin{tabular}{|c|c|c|c|c|c|}
\hline Study and Year & $\begin{array}{l}\text { Interventions } \\
\text { Comparisons }\end{array}$ & No. of Patients & DFS & OS & $\begin{array}{c}\text { Pattern of } \\
\text { First Relapse }\end{array}$ \\
\hline Ben-Josef et al, ${ }^{13} 2015$ & CRT (no comparison group) & 79 & $\begin{array}{l}\text { 2-year DFS: } 54 \% \\
\text { (95\% Cl, } 40 \% \text { to } \\
66 \%)\end{array}$ & $\begin{array}{l}\text { 2-year OS: } \\
65 \% \text { (95\% Cl, 53\% to } \\
74 \% \text { ) (exceeded } \\
\text { prespecified point } \\
\text { estimate threshold for } \\
\text { success of 45\%) } \\
\text { R0: } 67 \%(95 \% \mathrm{Cl}, 52 \% \\
\text { to } 78 \% \text { ) } \\
\text { R1: } 60 \%(95 \% \mathrm{Cl}, 38 \% \\
\text { to } 76 \% \text { ) (exceeded } \\
\text { prespecified point } \\
\text { estimate thresholds } \\
\text { of } 65 \% \text { and } 45 \% \text {, } \\
\text { respectively) }\end{array}$ & $\begin{array}{l}\text { Local: distal, 8\%; hilar, } \\
\text { 8\%; gallbladder, 0\% } \\
\text { Distant: distal, } 29 \% \text {; } \\
\text { hilar, 8\%; gallbladder, } \\
\text { 44\% } \\
\text { Local and distant: distal, } \\
\text { 13\%; hilar, 15\%; } \\
\text { gallbladder, } 8 \%\end{array}$ \\
\hline
\end{tabular}

Abbreviations: CRT, chemoradiotherapy; DFS, disease-free survival; OS, overall survival; RO, negative surgical margins ${ }^{14}$; R1, positive microscopic margins. ${ }^{14}$

with cholangiocarcinoma with $\mathrm{R} 1$ resections (odds ratio, $0.36 ; 95 \% \mathrm{Cl}, 0.14$ to $0.92 ; P=.03$ ). The authors also found a benefit of adjuvant radiation for all patients with biliary tract cancer with $\mathrm{R} 1$ resection (odds ratio, 0.33; 95\% $\mathrm{Cl}, 0.14$ to $0.81 ; P<.01$ ), whereas radiation therapy was not shown to be effective in R0 patients. ${ }^{8}$

Based on these data and the demonstrated higher rate of R1 resection in patients with hilar cholangiocarcinoma due to the location of the tumor, the Expert Panel agreed that adjuvant chemoradiation therapy may be offered for patients with hilar cholangiocarcinoma with R1 resections. Patients with extrahepatic and gallbladder cholangiocarcinoma with R1 resections are also included within Recommendation 2, because the Expert Panel agreed that the effect of chemoradiation would not be expected to differ by site and because the evidence contributing to this judgment included subsites other than hilar. The Expert Panel did not include patients with intrahepatic cholangiocarcinoma within this recommendation because there is no distinct margin to target with postoperative radiation for resected intrahepatic cholangiocarcinoma. The Expert Panel concluded that the competing risk of distant failure is high for patients with intrahepatic cholangiocarcinoma, and ablative radiation is a well-tolerated future option in the unlikely event that there is an isolated local failure.

Panel members concluded that adjuvant chemoradiation therapy may be a reasonable option to address the goal of reducing the risk of local recurrence. The Expert Panel recommends a shared decision-making approach considering the limited evidence base and including a discussion with the patient and relevant caregivers of the risks of harm and potential for benefit associated with adjuvant therapy. Further discussion of the consensus-based rationale for adjuvant therapy within this population is included in the Discussion.

\section{DISCUSSION}

Until recently, the evidence base for the treatment of resected biliary tract cancer consisted of mostly smaller retrospective studies with data combined for intrahepatic and extrahepatic cholangiocarcinoma and gallbladder cancer. Previous systematic reviews of retrospective studies have suggested a benefit of adjuvant therapy for patients with biliary tract cancer, ${ }^{38}$ a benefit of adjuvant radiation for extrahepatic cholangiocarcinoma, ${ }^{39}$ and an effect of adjuvant therapy in patients with biliary tract cancer with positive surgical margins and/or nodal involvement, ${ }^{8}$ with the caveat that randomized controlled trials are needed to confirm these exploratory results. This clinical practice guideline incorporates recently published data from prospective trials in biliary tract cancer and includes a review of retrospective studies for a subset of patients who are at higher risk for microscopically positive surgical margins.

Results from the BILCAP phase III randomized controlled trial of adjuvant capecitabine therapy in patients with biliary tract cancer were recently presented. This article is in press with Lancet Oncology. and showed a positive effect of adjuvant therapy on OS compared with observation (51.1 V 36.4 months, respectively) in adjusted ITT and per-protocol analyses, as well as a significant difference in RFS, while finding no significant difference in OS in an unadjusted ITT analysis. Based on these findings, the Expert Panel recommends adjuvant therapy with capecitabine for a period of 6 months. The Expert Panel noted that the dose of capecitabine $\left(1,250 \mathrm{mg} / \mathrm{m}^{2}\right.$ twice a day) may differ from the dose that is commonly given at some institutions in the United States and other locations outside of Europe. To allow for this practice variation, a qualifying statement that the recommended dose of capecitabine may be determined by institutional and regional practices was 


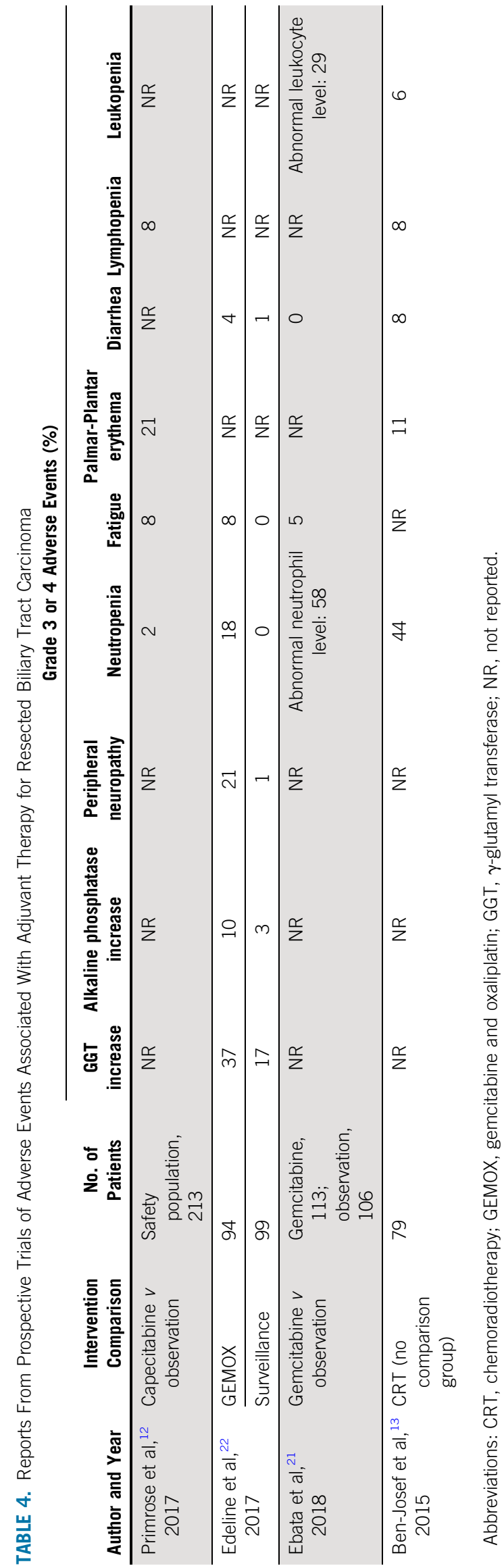


included. This is a moderate-strength recommendation, due to characteristics of the evidence base, such as the inclusion of only one randomized trial, inconsistency of the results between per-protocol and ITT analyses, and questions of generalizability to Asian populations. ${ }^{40}$ Nonetheless, based on the results of BILCAP, there is general agreement that this option should be offered to patients and that current and future randomized controlled trials should use capecitabine as the comparison arm. In response to this consensus, the ongoing ACTICCA-1 (Adjuvant Chemotherapy With Gemcitabine and Cisplatin Compared to Standard of Care After Curative Intent Resection of Biliary Tract Cancer) randomized controlled trial has changed its control arm from surveillance to capecitabine. ${ }^{40}$

No significant difference was detected in RFS or OS between the treatment and observation arms in other recently completed phase III randomized controlled trials of adjuvant gemcitabine (Ebata et $\mathrm{al}^{21}$ ) or gemcitabine and oxaliplatin (PRODIGE $12^{22}$ ). It is difficult to accrue patients with this uncommon diagnosis to randomized controlled trials, and in both negative trials, low event rates and/or small samples likely contributed to inadequate power to detect a difference between treatment and control groups, but no trend was observed in terms of OS benefit in either study.

The Expert Panel specified a review of studies of hilar cholangiocarcinoma in the guideline protocol because of the higher risk of $\mathrm{R} 1$ resection within this patient subgroup. A systematic search resulted in 16 studies that met the inclusion criteria, and data were extracted according to resection margin status when that information was available. Due to limitations of the evidence base, including small sample sizes and inconsistency of results, the Expert Panel's recommendation for adjuvant therapy for patients with positive microscopic resection margins was largely based on the informal consensus of the Expert Panel, as well as data showing positive outcomes associated with adjuvant therapy in the higher risk population of patients with $\mathrm{R} 1$ resections across disease subsites, including gallbladder and distal extrahepatic cholangiocarcinoma. Although patients with hilar tumors were the focus of the literature search and would compose the majority of patients affected by Recommendation 2, the Expert Panel chose to provide guidance for treatment of all patients with extrahepatic and gallbladder tumors with $\mathrm{R} 1$ resection because the effectiveness of adjuvant therapy would not be expected to differ by disease site.

Due to the current low quality of the evidence base, a shared decision-making approach was emphasized, including a patient-clinician discussion of the risks of harm and potential for benefit associated with treatment.

Further consensus-based recommendations for higher risk patients based on combination of lymph node positivity and margin status were considered to be outside the scope of this guideline. However, the Expert Panel noted a rationale for capecitabine chemotherapy in lymph node-positive patients with RO resections to control distant spread of disease, while patients with positive lymph nodes and positive surgical margins could benefit from capecitabine and chemoradiation therapy to control both local and distant recurrence. In addition, the Expert Panel considered that the order of implementation of Recommendations 1 and 2 would depend on the characteristics of individual patients and institutional practices.

The recommendations contained within this guideline incorporate the most recent evidence for treatment of biliary tract cancer, including evidence from the BILCAP study ${ }^{12}$ of capecitabine monotherapy, the first positive phase III randomized controlled trial in resected biliary tract cancer. In addition, important phase III data are provided in the Ebata et $\mathrm{al}^{21}$ and PRODIGE $12^{22}$ randomized controlled trials and the SWOG0809 phase II trial, ${ }^{13}$ which surpassed its predetermined threshold for efficacy and demonstrates the feasibility of conducting a national trial in this patient population. This guideline also provides an evidence- and consensus-based recommendation for patients with microscopically positive surgical margins. The results of the ongoing ACTICCA-1 randomized controlled trial ${ }^{40}$ of capecitabine compared with cisplatin plus gemcitabine are awaited to further inform evidence-based recommendations for biliary tract cancer. Results of the Japan Clinical Oncology Group 1202 study comparing adjuvant S-1 to surveillance in resected biliary tract cancer might also add information about the role of fluoropyrimidines in this setting. ${ }^{41}$ There is also a need for future research that focuses on high-risk patient subgroups and reports results for specific biliary tract subsites and/or specific molecular alterations. The Expert Panel members will continue to assess the currency of these recommendations and consider the need to update this guideline on an annual basis.

\section{PATIENT AND CLINICIAN COMMUNICATION}

For recommendations and strategies to optimize patientclinician communication, see "Patient-Clinician Communication: American Society of Clinical Oncology Consensus Guideline."42

\section{HEALTH DISPARITIES}

Although ASCO clinical practice guidelines represent expert recommendations on the best practices in disease management to provide the highest level of cancer care, it is important to note that many patients have limited access to medical care. Racial and ethnic disparities in health care contribute significantly to this problem in the United States. Patients with cancer who are members of racial or ethnic minorities suffer disproportionately from comorbidities, experience more substantial obstacles to receiving care, are more likely to be uninsured, and are at greater risk of receiving care of poor quality than other Americans. ${ }^{43-46}$ 
Many other patients lack access to care because of their geographic location and distance from appropriate treatment facilities. Awareness of these disparities in access to care should be considered in the context of this clinical practice guideline, and health care providers should strive to deliver the highest level of cancer care to these vulnerable populations.

\section{MULTIPLE CHRONIC CONDITIONS}

Creating evidence-based recommendations to inform treatment of patients with additional chronic conditions, a situation in which the patient may have two or more such conditions-referred to as multiple chronic conditions (MCCs) - is challenging. Patients with MCCs are a complex and heterogeneous population, making it difficult to account for all of the possible permutations to develop specific recommendations for care. In addition, the best available evidence for treating index conditions, such as cancer, is often from clinical trials whose study selection criteria may exclude these patients to avoid potential interaction effects or confounding of results associated with MCC. As a result, the reliability of outcome data from these studies may be limited, thereby creating constraints for expert groups to make recommendations for care in this heterogeneous patient population.

Because many patients to whom guideline recommendations apply present with MCCs, any treatment plan needs to take into account the complexity and uncertainty created by the presence of MCCs, highlighting the importance of shared decision making regarding guideline use and implementation. Therefore, in consideration of recommended care for the target index condition, clinicians should review all other chronic conditions present in the patient and take those conditions into account when formulating the treatment and follow-up plan.

In light of these considerations, practice guidelines should provide information on how to apply the recommendations for patients with MCCs, perhaps as a qualifying statement for recommended care. This may mean that some or all of the recommended care options are modified or not applied, as determined by best practice in consideration of any MCCs.

\section{COST IMPLICATIONS}

Increasingly, individuals with cancer are required to pay a larger proportion of their treatment costs through deductibles and coinsurance. ${ }^{47,48}$ Higher patient out-ofpocket costs have been shown to be a barrier to initiating and adhering to recommended cancer treatments. ${ }^{49,50}$

Discussion of cost can be an important part of shared decision making. ${ }^{51}$ Clinicians should discuss with patients the use of less expensive alternatives when it is practical and feasible for treatment of the patient's disease and there are two or more treatment options that are comparable in terms of benefits and harms. ${ }^{51}$

Patient out-of-pocket costs may vary depending on insurance coverage. Coverage may originate in the medical or pharmacy benefit, which may have different cost-sharing arrangements. Patients should be aware that different products may be preferred or covered by their particular insurance plan. Even with the same insurance plan, the price may vary between different pharmacies. When discussing financial issues and concerns, patients should be made aware of any financial counseling services available to address this complex and heterogeneous landscape. ${ }^{51}$

As part of the guideline development process, ASCO may opt to search the literature for published costeffectiveness analyses that might inform the relative value of available treatment options. Excluded from consideration are cost-effectiveness analyses that lack contemporary cost data, include agents that are not currently available in either the United States or Canada, and/or are industry sponsored. No cost-effectiveness analyses were identified to inform the topic.

\section{OPEN COMMENT}

The draft recommendations were released to the public for open comment from August 31, 2018, through September 14, 2018. Response categories of "Agree as written," "Agree with suggested modifications," and "Disagree. See comments" were captured for every proposed recommendation with three written comments received. Two of the three respondents either agreed or agreed with slight modifications to the recommendations, and one respondent disagreed with Recommendation 1. Expert Panel members reviewed comments from all sources and determined whether to maintain original draft recommendations, revise with minor changes, or consider major recommendation revisions. All changes were incorporated before Clinical Practice Guidelines Committee review and approval.

\section{GUIDELINE IMPLEMENTATION}

ASCO guidelines are developed for implementation across health settings. Barriers to implementation include the need to increase awareness of the guideline recommendations among front-line practitioners and survivors of cancer and caregivers and also to provide adequate services in the face of limited resources. The guideline Bottom Line box was designed to facilitate implementation of recommendations. This guideline will be distributed widely through the ASCO Practice Guideline Implementation Network. ASCO guidelines are posted on the ASCO Web site and most often published in Journal of Clinical Oncology and Journal of Oncology Practice.

ASCO believes that cancer clinical trials are vital to inform medical decisions and improve cancer care 
and that all patients should have the opportunity to participate.

\section{ADDITIONAL RESOURCES}

More information, including a Data Supplement with additional evidence tables, slide sets, and clinical tools and resources, is available at www.asco.org/gastrointestinal-cancerguidelines. The Methodology Manual (available at www.asco. org/guideline-methodology) provides additional information about the methods used to develop this guideline. Patient information is available at www.cancer.net.

\section{AFFILIATIONS}

${ }^{1}$ University of Arizona Cancer Center, Tucson, AZ

${ }^{2}$ American Society of Clinical Oncology, Alexandria, VA

${ }^{3}$ Cholangiocarcinoma Foundation, Billings, MO

${ }^{4}$ Mayo Clinic, Phoenix, AZ

${ }^{5}$ Memorial Sloan Kettering Cancer Centre, New York, NY

${ }^{6}$ Centre Eugène Marquis, Rennes, France

${ }^{7}$ University of Southern California Kenneth Norris Cancer Center, Los Angeles, CA

${ }^{8}$ University of California, San Francisco, CA

${ }^{9}$ The University of Texas MD Anderson Cancer Center, Houston, TX

${ }^{10}$ University of Southampton, Southampton, United Kingdom

${ }^{11}$ University of New Mexico Comprehensive Cancer Center, Albuquerque, NM

${ }^{12}$ University of Manchester Institute of Cancer Sciences, Manchester, United Kingdom

${ }^{13}$ Winship Cancer Institute, Emory University, Atlanta, GA

\section{RELATED ASCO GUIDELINES}

- Integration of Palliative Care Into Standard Oncology Practice (http://ascopubs.org/doi/ 10.1200/JCO.2016.70.1474) $)^{52}$

- Patient-Clinician Communication (http://ascopubs. org/doi/10.1200/JCO.2017.75.2311) ${ }^{42}$

\section{CORRESPONDING AUTHOR}

American Society of Clinical Oncology, 2318 Mill Rd, Ste 800, Alexandria, VA 22314; e-mail: guidelines@asco.org.

\section{AUTHORS' DISCLOSURES OF POTENTIAL CONFLICTS OF INTEREST AND DATA AVAILABILITY STATEMENT}

Disclosures provided by the authors and data availability statement (if applicable) are available with this article at DOI https://doi.org/10.1200/ JCO.18.02178

\section{AUTHOR CONTRIBUTIONS}

Manuscript writing: All authors

Final approval of manuscript: All authors

Accountable for all aspects of the work: All authors

\section{ACKNOWLEDGMENT}

The Expert Panel thanks Dr. Jeremy Kortmansky, MD, and Dr. Nishin A. Bhadkamkar, MD, and the Clinical Practice Guidelines Committee for their thoughtful reviews and insightful comments on this guideline.

\section{REFERENCES}

1. American Cancer Society: Cancer Facts \& Figures 2018. https://www.cancer.org/content/dam/cancer-org/research/cancer-facts-and-statistics/annual-cancerfacts-and-figures/2018/cancer-facts-and-figures-2018.pdf

2. American Cancer Society: Bile duct cancer stages. https://www.cancer.org/cancer/bile-duct-cancer/detection-diagnosis-staging/staging.html

3. Hundal R, Shaffer EA: Gallbladder cancer: Epidemiology and outcome. Clin Epidemiol 6:99-109, 2014

4. Nakeeb A, Pitt HA, Sohn TA, et al: Cholangiocarcinoma: A spectrum of intrahepatic, perihilar, and distal tumors. Ann Surg 224:463-473, discussion 473-475, 1996

5. National Comprehensive Cancer Network: Hepatobiliary cancers. https://www.nccn.org/professionals/physician_gls/default.aspx

6. Bridgewater JA, Goodman KA: Biliary tract cancer: Epidemiology, radiotherapy, and molecular profiling. Am Soc Clin Oncol Educ Book 35:e194-e203, 2016

7. Nyberg K: French phase III trial reveals adjuvant GEMOX no better than surveillance in localized biliary tract cancer. https://gicasym.org/daily-news/frenchphase-iii-trial-reveals-adjuvant-gemox-no-better-surveillance-localized-biliary

8. Horgan AM, Amir E, Walter T, et al: Adjuvant therapy in the treatment of biliary tract cancer: A systematic review and meta-analysis. J Clin Oncol 30:1934-1940, 2012

9. Valle JW, Borbath I, Khan SA, et al: Biliary cancer: ESMO Clinical Practice Guidelines for diagnosis, treatment and follow-up. Ann Oncol 27:v28-v37, 2016 (suppl 5)

10. Valle JW, Borbath I, Khan SA, et al: Biliary cancer: ESMO Clinical Practice Guidelines for diagnosis, treatment and follow-up. Ann Oncol 27:v28-v37, 2016 (suppl 5)

11. Mansour JC, Aloia TA, Crane CH, et al: Hilar cholangiocarcinoma: Expert consensus statement. HPB 17:691-699, 2015

12. Primrose JN, Fox R, Palmer DH, et al: Adjuvant capecitabine for biliary tract cancer: The BILCAP randomized study. J Clin Oncol 35, 2017 (suppl 15; abstr 4006)

13. Ben-Josef E, Guthrie KA, El-Khoueiry AB, et al: SWOG S0809: A phase II intergroup trial of adjuvant capecitabine and gemcitabine followed by radiotherapy and concurrent capecitabine in extrahepatic cholangiocarcinoma and gallbladder carcinoma. J Clin Oncol 33:2617-2622, 2015

14. Hermanek P, Wittekind C: Residual tumor (R) classification and prognosis. Semin Surg Oncol 10:12-20, 1994

15. Salem ME, Kaseb AO, Krishnan S: Using radiation therapy in resected biliary tract cancer. https://gicasym.org/daily-news/using-radiation-therapy-resectedbiliary-tract-cancer

16. Shiffman RN, Michel G, Rosenfeld RM, et al: Building better guidelines with BRIDGE-Wiz: Development and evaluation of a software assistant to promote clarity, transparency, and implementability. J Am Med Inform Assoc 19:94-101, 2012

17. Cochrane Collaboration: Cochrane Risk of Bias Tool. https://methods.cochrane.org/bias/assessing-risk-bias-included-studies\#The\%20Cochrane\%20Risk\% 20of\%20Bias\%20Tool 
18. Higgins JPT, Green S (eds): 12.2 Assessing the quality of a body of evidence, in Cochrane Handbook for Systematic Reviews of Interventions (volume 5.1.0). New York, NY, John Wiley \& Sons, 2011, pp 361-366

19. Schünemann HJ, Cuello C, AkI EA, et al: GRADE guidelines: 18. How ROBINS-I and other tools to assess risk of bias in nonrandomized studies should be used to rate the certainty of a body of evidence. J Clin Epidemiol 10.1016/j.jclinepi.2018.01.012 [Epub ahead of print on February 9, 2018]

20. Shojania KG, Sampson M, Ansari MT, et al: How quickly do systematic reviews go out of date? A survival analysis. Ann Intern Med 147:224-233, 2007

21. Ebata T, Hirano S, Konishi M, et al: Randomized clinical trial of adjuvant gemcitabine chemotherapy versus observation in resected bile duct cancer. Br J Surg 105:192-202, 2018

22. Edeline J, Bonnetain F, Phelip JM, et al: Adjuvant GEMOX for biliary tract cancer: Updated relapse-free survival and first overall survival results of the randomized PRODIGE 12-ACCORD 18 (UNICANCER GI) phase III trial. Ann Oncol 28:v605-v649, 2017 (suppl 5; abstr LBA29)

23. Baton O, Azoulay D, Adam DV, et al: Major hepatectomy for hilar cholangiocarcinoma type 3 and 4: Prognostic factors and longterm outcomes. J Am Coll Surg 204:250-260, 2007

24. Chauhan A, House MG, Pitt HA, et al: Post-operative morbidity results in decreased long-term survival after resection for hilar cholangiocarcinoma. HPB 13: 139-147, 2011

25. Cheng Q, Luo X, Zhang B, et al: Predictive factors for prognosis of hilar cholangiocarcinoma: Postresection radiotherapy improves survival. Eur J Surg Oncol 33: 202-207, 2007

26. Ecker BL, Vining CC, Roses RE, et al: Identification of patients for adjuvant therapy after resection of carcinoma of the extrahepatic bile ducts: A propensity score-matched analysis. Ann Surg Oncol 24:3926-3933, 2017

27. Higuchi R, Ota T, Yazawa T, et al: Improved surgical outcomes for hilar cholangiocarcinoma: Changes in surgical procedures and related outcomes based on 40 years of experience at a single institution. Surg Today 46:74-83, 2016

28. Im JH, Seong J, Lee IJ, et al: Surgery alone versus surgery followed by chemotherapy and radiotherapy in resected extrahepatic bile duct cancer: Treatment outcome analysis of 336 patients. Cancer Res Treat 48:583-595, 2016

29. Kobayashi H, Murakami $\mathrm{Y}$, Uemura $\mathrm{K}$, et al: Human equilibrative nucleoside transporter 1 expression predicts survival of advanced cholangiocarcinoma patients treated with gemcitabine-based adjuvant chemotherapy after surgical resection. Ann Surg 256:288-296, 2012

30. Krasnick BA, Jin LX, Davidson JT IV, et al: Adjuvant therapy is associated with improved survival after curative resection for hilar cholangiocarcinoma: A multiinstitution analysis from the U.S. extrahepatic biliary malignancy consortium. J Surg Oncol 117:363-371, 2018

31. Leng KM, Liu YP, Wang ZD, et al: Results of adjuvant radiation therapy for locoregional perihilar cholangiocarcinoma after curative intent resection. Onco Targets Ther 10:2257-2266, 2017

32. Mizuno T, Ebata T, Yokoyama Y, et al: Adjuvant gemcitabine monotherapy for resectable perihilar cholangiocarcinoma with lymph node involvement: $\mathrm{A}$ propensity score matching analysis. Surg Today 47:182-192, 2017

33. Murakami Y, Uemura K, Sudo T, et al: Gemcitabine-based adjuvant chemotherapy improves survival after aggressive surgery for hilar cholangiocarcinoma. J Gastrointest Surg 13:1470-1479, 2009

34. Nassour I, Mokdad AA, Porembka MR, et al: Adjuvant therapy is associated with improved survival in resected perihilar cholangiocarcinoma: A propensity matched study. Ann Surg Oncol 25:1193-1201, 2018

35. Sagawa N, Kondo S, Morikawa T, et al: Effectiveness of radiation therapy after surgery for hilar cholangiocarcinoma. Surg Today 35:548-552, 2005

36. Wirasorn K, Ngamprasertchai T, Khuntikeo N, et al: Adjuvant chemotherapy in resectable cholangiocarcinoma patients. J Gastroenterol Hepatol 28:1885-1891, 2013

37. Zervos EE, Osborne D, Goldin SB, et al: Stage does not predict survival after resection of hilar cholangiocarcinomas promoting an aggressive operative approach. Am J Surg 190:810-815, 2005

38. Ghidini M, Tomasello G, Botticelli A, et al: Adjuvant chemotherapy for resected biliary tract cancers: A systematic review and meta-analysis. HPB 19:741-748, 2017

39. Bonet Beltrán M, Allal AS, Gich I, et al: Is adjuvant radiotherapy needed after curative resection of extrahepatic biliary tract cancers? A systematic review with a meta-analysis of observational studies. Cancer Treat Rev 38:111-119, 2012

40. London S: BILCAP: Adjuvant capecitabine boosts overall survival of biliary tract cancers. https://www.mdedge.com/oncologypractice/article/138518/ gastroenterology/bilcap-adjuvant-capecitabine-boosts-overall

41. Nakachi K, Konishi M, Ikeda M, et al: A randomized phase III trial of adjuvant S-1 therapy vs. observation alone in resected biliary tract cancer: Japan Clinical Oncology Group study (JCOG1202, ASCOT). Jpn J Clin Oncol 48:392-395, 2018

42. Gilligan T, Coyle N, Frankel RM, et al: Patient-clinician communication: American Society of Clinical Oncology consensus guideline. J Clin Oncol 35:3618-3632, 2017

43. Mead H, Cartwright-Smith L, Jones K, et al: Racial and Ethnic Disparities in U.S. Health Care: A Chartbook. New York, NY, The Commonwealth Fund, 2008

44. US Cancer Statistics Working Group: United States cancer statistics: 1999-2012 incidence and mortality Web-based report. https://www.cdc.gov/cancer/uscs/

45. Howlader N, Noone AM, Krapcho M, et al: SEER Cancer Statistics Review, 1975-2013. https://seer.cancer.gov/archive/csr/1975_2013/

46. American Cancer Society: Cancer facts and figures for African Americans 2016-2018. http://www.cancer.org/acs/groups/content/@editorial/documents/ document/acspc-047403.pdf

47. Schnipper LE, Davidson NE, Wollins DS, et al: Updating the American Society of Clinical Oncology Value Framework: Revisions and reflections in response to comments received. J Clin Oncol 34:2925-2934, 2016

48. Schnipper LE, Davidson NE, Wollins DS, et al: American Society of Clinical Oncology statement: A conceptual framework to assess the value of cancer treatment options. J Clin Oncol 33:2563-2577, 2015

49. Dusetzina SB, Winn AN, Abel GA, et al: Cost sharing and adherence to tyrosine kinase inhibitors for patients with chronic myeloid leukemia. J Clin Oncol 32: 306-311, 2014

50. Streeter SB, Schwartzberg L, Husain N, et al: Patient and plan characteristics affecting abandonment of oral oncolytic prescriptions. J Oncol Pract 7:46s-51s, 2011 (suppl 3)

51. Meropol NJ, Schrag D, Smith TJ, et al: American Society of Clinical Oncology guidance statement: The cost of cancer care. J Clin Oncol 27:3868-3874, 2009

52. Ferrell BR, Temel JS, Temin S, et al: Integration of palliative care into standard oncology care: American Society of Clinical Oncology clinical practice guideline update. J Clin Oncol 35:96-112, 2017 


\section{Adjuvant Therapy for Resected Biliary Tract Cancer: ASCO Clinical Practice Guideline}

The following represents disclosure information provided by authors of this manuscript. All relationships are considered compensated. Relationships are self-held unless noted. I = Immediate Family Member, Inst = My Institution. Relationships may not relate to the subject matter of this manuscript. For more information about ASCO's conflict of interest policy, please refer to www.asco.org/rwc or ascopubs.org/jco/site/ifc.

Rachna T. Shroff

Consulting or Advisory Role: Halozyme, Seattle Genetics, Exelixis Research Funding: Eli Lilly, Celgene, Agios, Halozyme

Travel, Accommodations, Expenses: Halozyme, Seattle Genetics

Melinda Bachini

Honoraria: Incyte

Consulting or Advisory Role: Incyte

Travel, Accommodations, Expenses: Incyte, Bayer

Other Relationship: Taiho Pharmaceutical, QED

Tanios Bekaii-Saab

Consulting or Advisory Role: Amgen (Inst), Glenmark, Ipsen (Inst), Eli Lilly (Inst), Bristol-Myers Squibb (Inst), Merck (Inst), Bayer (Inst), Celgene (Inst), Genentech (Inst), Abbvie, Incyte (Inst), Imugene, Immuneering

Other Relationship: Exelixis, Merck, Sillajen, ARMO BioSciences

\section{Christopher Crane}

Honoraria: Celgene

\section{Julien Edeline}

Consulting or Advisory Role: BTG, Bristol-Myers Squibb, AstraZeneca, Bayer, Ipsen, AstraZeneca

Research Funding: Bristol-Myers Squibb (Inst)

Travel, Accommodations, Expenses: Amgen, Bristol-Myers Squibb

\section{Anthony El-Khoueiry}

Honoraria: Bayer, Novartis, Bristol-Myers Squibb, Genentech, EMD Serono, Eisai, Merck

Consulting or Advisory Role: CytomX Therapeutics, Bristol-Myers Squibb, AstraZeneca, Bayer, Eisai, Roche, EMD Serono, Merck, Exelixis

Research Funding: AstraZeneca, Astex Pharmaceuticals
Mary Feng

Honoraria: Medivation/Astellas (I), Myriad Pharmaceuticals (I), Reflexion Medical

Consulting or Advisory Role: GenomeDx (I), NanoString Technologies (I), Myriad Pharmaceuticals (I), Varian Medical Systems

Speakers' Bureau: Medivation/Astellas (I)

Research Funding: Celgene (I), Varian Medical Systems (Inst)

Patents, Royalties, Other Intellectual Property: Patent pending for RadioType Dx, a biomarker test (I)

Travel, Accommodations, Expenses: GenomeDx (I)

\section{Matthew H.G. Katz}

Consulting or Advisory Role: Alcresta Therapeutics, Abbvie

Heloisa P. Soares

Consulting or Advisory Role: Ipsen, Cornerstone Pharmaceuticals, Bayer,

Exelixis, Lexicon, Celgene

Speakers' Bureau: Celgene, Novartis

\section{Juan Valle}

Honoraria: Ipsen

Consulting or Advisory Role: Ipsen, Novartis, AstraZeneca, Merck, Delcath Systems, Agios, Pfizer, PCl Biotech, Incyte, Keocyt, QED, Pieris

Pharmaceuticals, Genoscience Pharma

Speakers' Bureau: Novartis, Ipsen

Research Funding: Novartis (Inst)

Travel, Accommodations, Expenses: Celgene, Nucana

No other potential conflicts of interest were reported. 


\section{APPENDIX}

TABLE A1. Guideline Expert Panel Members Name and Designation

\begin{tabular}{lll}
\hline Rachna T. Shroff, MD, MS (co-chair) & University of Arizona Cancer Center, Tucson, AZ & Medical oncology \\
\hline Shishir K. Maithel, MD (co-chair) & Emory Winship Cancer Institute, Atlanta, GA & Surgical oncology \\
\hline Anthony El-Khoueiry, MD & $\begin{array}{c}\text { University of Southern California Kenneth Norris Cancer } \\
\text { Center, Los Angeles, CA }\end{array}$ & Medical oncology \\
\hline Tanios Bekaii-Saab, MD & Mayo Clinic, Phoenix, AZ & Medical oncology \\
\hline Julien Edeline, MD, PhD & Centre Eugène Marquis, Rennes, France & Medical oncology \\
\hline Juan Valle, MD, ChB, MSc & University of Manchester Institute of Cancer Sciences, & Medical oncology \\
& Manchester, United Kingdom & Surgical oncology \\
\hline John Primrose, MD, ChB & University of Southampton, Southampton, United & Radiation oncology \\
\hline Christopher Crane, MD & Memorial Sloan Kettering Cancer Center, New York, NY & Radiation oncology \\
\hline Mary Feng, MD & University of California, San Francisco, CA & Surgical oncology \\
\hline Matthew H.G. Katz, MD & The University of Texas MD Anderson Cancer Center, & \\
\hline Melinda Bachini & Houston, TX & Patient representative \\
\hline Heloisa P. Soares, MD, PhD & Bdvocacy Coordinator, Cholangiocarcinoma Foundation, MO & Practice Guidelines Implementation Network \\
\hline Erin B. Kennedy, MHSc & University of New Mexico Comprehensive Cancer Center, & \\
\hline
\end{tabular}

